\title{
Expression of GPR17, a regulator of oligodendrocyte differentiation and maturation, in Nasu-Hakola disease brains
}

\author{
Jun-ichi Satoh $^{1, *}$, Yoshihiro Kino ${ }^{1}$, Motoaki Yanaizu ${ }^{1}$, Youhei Tosaki ${ }^{1}$, Kenji Sakai ${ }^{1}$, \\ Tusyoshi Ishida ${ }^{2}$, Yuko Saito ${ }^{3}$ \\ ${ }^{1}$ Department of Bioinformatics and Molecular Neuropathology, Meiji Pharmaceutical University, Tokyo, Japan; \\ ${ }^{2}$ Department of Pathology and Laboratory Medicine, Kohnodai Hospital, NCGM, Chiba, Japan; \\ ${ }^{3}$ Department of Laboratory Medicine, National Center Hospital, NCNP, Tokyo, Japan.
}

\begin{abstract}
Summary The G protein-coupled receptor 17 (GPR17), a Gi-coupled GPCR, acts as an intrinsic timer of oligodendrocyte differentiation and myelination. The expression of GPR17 is upregulated during differentiation of oligodendrocyte precursor cells (OPCs) into premyelinating oligodendrocytes (preoligodendrocytes), whereas it is markedly downregulated during terminal maturation of myelinating oligodendrocytes. Nasu-Hakola disease (NHD) is a rare autosomal recessive disorder caused by a loss-of-function mutation of either TYROBP (DAP12) or TREM2. Pathologically, the brains of NHD patients exhibit extensive demyelination designated leukoencephalopathy, astrogliosis, accumulation of axonal spheroids, and activation of microglia predominantly in the white matter of frontal and temporal lobes. Although GPR17 is a key regulator of oligodendrogenesis, a pathological role of GPR17 in NHD brains with relevance to development of leukoencephalopathy remains unknown. We studied the expression of GPR17 in five NHD brains and eight control brains by immunohistochemistry. We identified GPR17-immunoreactive preoligodendrocytes with a multipolar ramified morphology distributed in the white matter and the grey matter of all cases examined. However, we did not find statistically significant differences in the number of GPR17-expressing cells between NHD and control brains both in the white matter and the grey matter due to great variability from case to case. These observations do not support the view that GPR17-positive preoligodendrocytes play a central role in the development of leukoencephalopathy in NHD brains.
\end{abstract}

Keywords: GPR17, leukoencephalopathy, Nasu-Hakola disease, oligodendrocytes, preoligodendrocytes

\section{Introduction}

The G protein-coupled receptor 17 (GPR17), a Gi-coupled GPCR, acts as an intrinsic timer of oligodendrocyte differentiation and myelination $(1,2)$. In the central nervous system (CNS), GPR17 is expressed in the oligodendrocyte lineage cells, and its levels

Released online in J-STAGE as advance publication February $14,2017$.

*Address correspondence to:

Dr. Jun-ichi Satoh, Department of Bioinformatics and Molecular Neuropathology, Meiji Pharmaceutical University, 2-522-1 Noshio, Kiyose, Tokyo, Japan.

E-mail: satoj@my-pharm.ac.jp are elevated during differentiation of NG2-positive oligodendrocyte precursor cells (OPCs) into O4-positive premyelinating oligodendrocytes (preoligodendrocytes), whereas it is markedly downregulated during terminal maturation of myelinating oligodendrocytes that express myelin basic protein (MBP) and 2',3'-cyclicnucleotide 3'-phosphodiesterase (CNPase), suggesting that GPR17 serves as a valid marker to identify an intermediate phase of OPC differentiation (1-3). Phylogenetically, GPR17 is located at an intermediate position between purinergic $\mathrm{P} 2 \mathrm{Y}$ receptor and cysteinyl leukotriene (CysLT) receptor (4). GPR17 recognizes two distinct types of endogenous ligands, including uracil nucleotides, such as uridine diphosphate (UDP) and UDP-glucose and CysLTs, such as leukotriene D4 
$\left(\mathrm{LTD}_{4}\right)$ and leukotriene $\mathrm{E} 4\left(\mathrm{LTE}_{4}\right)(4)$. The endogenous ligands for GPR17 are released extracellularly from damaged cells at sites of trauma, ischemia, and inflammation, where GPR17 expression is upregulated markedly around the lesions, suggesting that GPR17 serves as a sensor of brain damage $(2,5)$.

GPR17 transgenic mice under the control of the CNP promoter show generalized tremors, hindlimb paralysis, and seizure due to a defect in myelinogenesis by blockade of oligodendrocyte maturation (1). GRP17 overexpression induces nuclear localization of differentiation inhibitors, such as ID2 and ID4, which in turn inhibit oligodendrocyte differentiation and maturation by sequestrating Olig1, the basic helixloop-helix (bHLH) transcription factor essential for oligodendrocyte differentiation and myelination (1). In contrast, GPR17 knockout mice reveal accelerated differentiation of OPCs with an early onset of myelination (1). These observations suggest that GPR17 acts as a negative regulator of oligodendrocyte differentiation and maturation.

Importantly, GPR 17 activation and inactivation by natural or synthetic agonists and antagonists show different effects on OPC differentiation and maturation. The exposure of OPCs to GPR17 endogenous ligands, such as UDP glucose and $\mathrm{LTD}_{4}$, promotes differentiation of preoligodendrocytes to mature myelinating oligodendrocytes (5). In OPCs and preoligodendrocytes, GPR17 stimulation by UDP glucose enhances outward $\mathrm{K}^{+}$currents responsible for GPR17-induced maturation and migration (6). In contrast, GPR17 activation in differentiating oligodendrocytes by synthetic agonist MDL29,951 downregulates MBP expression by reducing the activity of the adenyl cyclase-cyclic adenosine monophosphate (cAMP)-protein kinase A (PKA)-cAMP response element-binding protein (CREB) cascade (7). On the contrary, GPR17 inhibition by an antagonist cangrelor or siRNAs, inhibits OPC differentiation and maturation (3). The apparently inconsistent results are partly derived from distinct pathophysiological conditions examined, availability of endogenous ligands, and the stage-specific roles of GPR17, that is a positive role for differentiation in early OPCs and a negative role for oligodendrocyte maturation in late OPCs (2). In addition, GPR17-mediated responses are changeable, depending on the heterodimerization with other GPCR receptors, such as $\mathrm{CysLT}_{1} \mathrm{R}(8)$. Furthermore, internalization, degradation, recycling, and desensitization of GPR17 regulate GPR17-mediated signaling pathway $(2,9)$. Thus, GPR17 plays a complex role in regulation of oligodendrocyte differentiation and maturation. OPCs are present in demyelinating lesions of multiple sclerosis (MS), where GRP17 expression is upregulated substantially but they fail to differentiate into mature oligodendrocytes $(1,10)$. It remains unknown whether an enhanced expression of GPR17 is beneficial or detrimental for OPC differentiation, maturation, and myelination in adult human brain diseases.

Nasu-Hakola disease (NHD), also designated polycystic lipomembranous osteodysplasia with sclerosing leukoencephalopathy (PLOSL), is a rare autosomal recessive disorder, characterized by progressive presenile dementia and formation of multifocal bone cysts, caused by genetic mutations of either TYROBP (DAP12) or TREM2 (11). TREM2 and DAP12 constitute a receptor/adaptor signaling complex expressed exclusively on osteoclasts, dendritic cells, macrophages, and microglia. Although NHD patients are clustered in Japan and Finland, approximately 200 NHD cases are presently reported worldwide. Clinically, the patients with NHD show recurrent bone fractures during the third decade of life, and a frontal lobe syndrome during the fourth decade of life, and progressive dementia and death until the fifth decade of life (12). Pathologically, the brains of NHD patients exhibit extensive demyelination designated leukoencephalopathy, astrogliosis, accumulation of axonal spheroids, and remarkable activation of microglia predominantly in the white matter of frontal and temporal lobes and the basal ganglia (13). At present, molecular mechanisms responsible for development of leukoencephaolpathy in NHD brains remain totally unknown. Since GPR17 is a key regulator of oligodendrogenesis, we propose the hypothesis that progression of leukoencephalopathy in NHD brains is attributable to a failure of oligodendrocyte differentiation and maturation, possibly associated with aberrant expression of GPR17 on the oligodendrocytelineage cells. In the present study, we have attempted to clarify the expression of GPR17 in NHD brains by immunohistochemistry.

\section{Materials and Methods}

\subsection{Human brain tissues}

The brain autopsies were performed at the National Center Hospital, National Center of Neurology and Psychiatry (NCNP), Japan, Kohnodai Hospital, National Center for Global Health and Medicine (NCGM), Japan, and affiliated hospitals of Research Resource Network (RRN), Japan. The comprehensive examination by established neuropathologists (YS and $\mathrm{TI}$ ) validated the pathological diagnosis. Written informed consent was obtained in all cases. The Ethics Committee of the NCNP for the Human Brain Research, the Ethics Committee of the NCGM on the Research Use of Human Samples, and the Human Research Ethics Committee (HREC) of the Meiji Pharmaceutical University (MPU) approved the present study.

For immunohistochemical studies, paraffin sections of the cerebral cortex were prepared from four subjects who died of non-neurological causes (NC), composed of a 63-year-old man who died of prostate cancer and acute 
myocardial infarction (NC1), a 67-year-old man who died of dissecting aortic aneurysm (NC2), a 57-yearold man who died of alcoholic liver cirrhosis (NC3), and a 61-year-old man who died of rheumatoid arthritis with interstitial pneumonia (NC4), four neuropsychiatric disease controls affected with myotonic dystrophy (MD), composed of a 68-year-old man (MD1), a 61-year-old man (MD2), a 60-year-old man (MD3), and a 53-yearold woman (MD4), and five NHD patients, composed of a 42-year-old man (NHD1), a 48-year-old woman (NHD2), a 44-year-old man (NHD3), a 32-year-old woman (NHD4), and a 38-year-old man (NHD5). The homozygous mutation of a single base deletion of $141 \mathrm{G}$ (c.141delG) in exon 3 of DAP12 was identified in NHD1, NHD2, and NHD5, while the genetic analysis was not performed in NHD3 or NHD4, as described previously (14).

\subsection{Immunohistochemistry}

After deparaffination, tissue sections were heated in $10 \mathrm{mM}$ citrate sodium buffer, $\mathrm{pH} 6.0$ by autoclave at $110^{\circ} \mathrm{C}$ for $15 \mathrm{~min}$ in a temperature-controlled pressure chamber (Biocare Medical, Concord, CA, USA). They were treated at room temperature (RT) for 15 min with $3 \%$ hydrogen peroxide-containing methanol to block the endogenous peroxidase activity. They were then incubated with phosphate-buffered saline (PBS) containing 10\% normal goat serum at RT for 15 min to block non-specific staining, followed by incubation in a moist chamber at $4^{\circ} \mathrm{C}$ overnight with rabbit polyclonal anti-GPR17 antibody (HPA029766, Sigma, St. Louis, MO, USA). After washing with PBS, tissue sections were incubated at RT for $30 \mathrm{~min}$ with the horseradish peroxidase (HRP)-conjugated anti-rabbit secondary antibody (Nichirei, Tokyo, Japan), followed by incubation with diaminobenzidine tetrahydrochloride (DAB) substrate (Vector, Burlingame, CA, USA). They were processed for a counterstain with hematoxylin. Negative controls underwent all the steps except for exposure to primary antibody. In limited experiments, double immunolabeling of rabbit anti-GPR17 antibody with mouse monoclonal antibodies against GFAP (GA5, Nichirei) for astrocytes, gp91phox (ab139371, Abcam, Cambridge, UK) for microglia (14), NeuN (ab104224, Abcam) for neurons, NG2 (LHM2, Novus Biologicals, Littleton, CO, USA) for early OPCs, O4 (a gift from Dr. Seung U. Kim, University of British Columbia) for preoligodendrocytes, or CNPase (11-5B, Sigma) and MBP (ab62631, Abcam) for mature oligodendrocytes, was performed, followed by incubation with HRPconjugated or alkaline phosphatase-conjugated antirabbit or anti-mouse secondary antibody and exposure to DAB substrate and Warp Red chromogen (Biocare Medical).

\subsection{Quantification of GPR17 immunoreactive cells}

The number of GPR17-positive cells in ten fields of the white matter or the grey matter were manually counted at a $200 \times$ magnification on the Olympus BX51 universal microscope. The difference in the average of immunopositive cell numbers between NHD and the controls was evaluated by one-way analysis of variance (ANOVA) followed by post-hoc Tukey's test.

\section{Results and Discussion}

By immunohistochemistry, we identified GPR17immunoreactive cells distributed in the white matter and the grey matter in all cases of NC, MD, and NHD brains. The virtually all of GPR17-immunoreactive cells showed a multipolar ramified morphology corresponding to preoligodendrocytes (3) (Figure 1, panels a-d for NC, Figure 2, panels a-d for MD, and Figure 3, panels a-d for NHD). They did not express

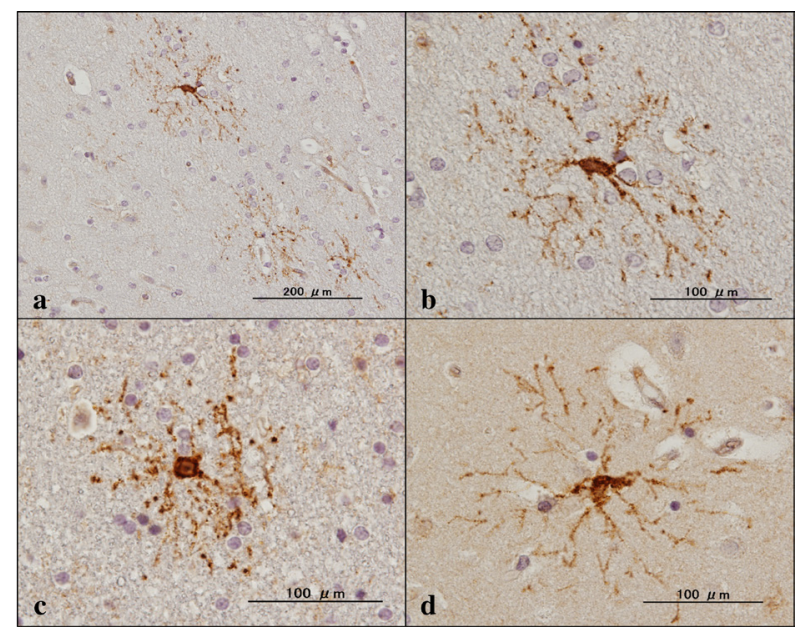

Figure 1. Expression of GPR17 in NC brains. GRP17immuoreactive ramified cells in (a) the white matter (NC2), (b) the higher magnification photograph of (a), (c) the white matter (NC3), and (d) the grey matter (NC3).

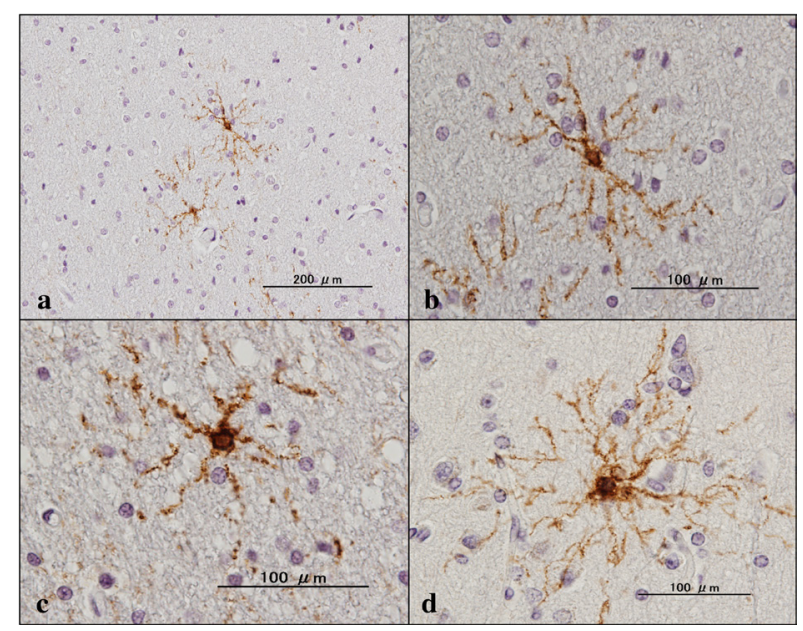

Figure 2. Expression of GPR17 in MD brains. GRP17immuoreactive ramified cells in (a) the white matter (MD4), (b) the higher magnification photograph of (a), (c) the white matter (MD4), and (d) the grey matter (MD1). 


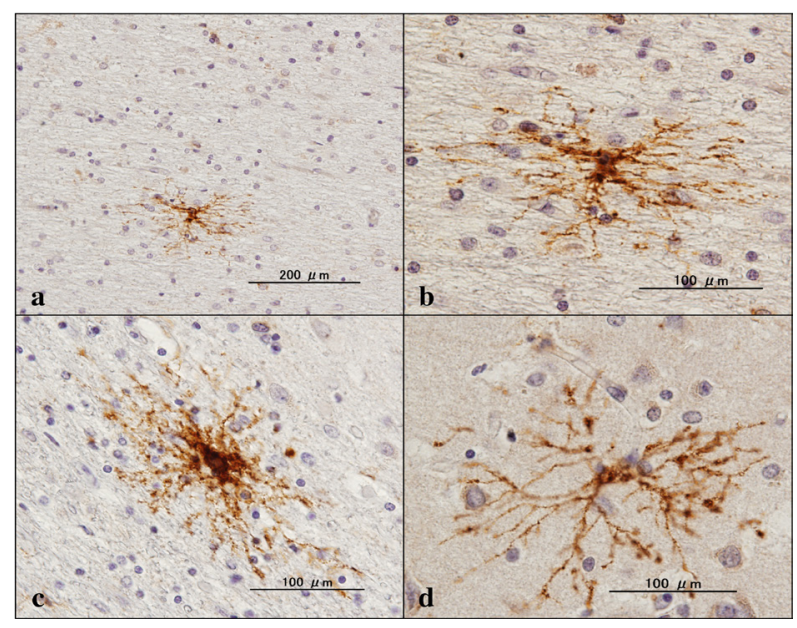

Figure 3. Expression of GPR17 in NHD brains. GRP17immuoreactive ramified cells in (a) the demyelinated white matter (NHD4), (b) the higher magnification photograph of (a), (c) the demyelinated white matter (NHD2), and (d) the grey matter (NHD2).

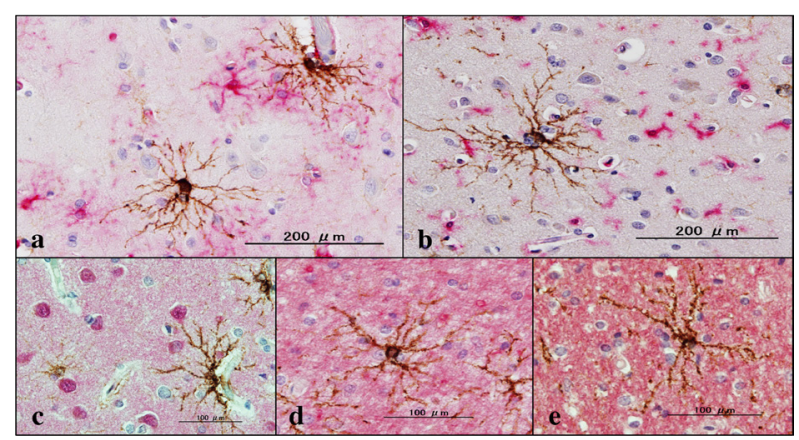

Figure 4. Double immunolabeling of GPR17 with cell type-specific markers. Double labeling of GRP17 (brown) with (a) GFAP (red), (b) gp91phox (red), (c) NeuN (red), (d) CNP (red), and (e) MBP (red). All derived from MD4.

GFAP, gp91phox, NeuN, CNPase, or MBP, indicating that they are not astrocytes, microglia, neurons, or mature oligodendrocytes (Figure 4, panels a-e). We could not label the cells with mouse monoclonal antibodies against NG2 or O4, a maker for OPCs and preoligodendrocytes, because they seem to be only applicable for frozen tissues that are unavailable in the present study. We found considerable variability in the number of GRP17-positive cells from case to case and between the white matter and the grey matter of NC, MD, and NHD brains. Therefore, we did not identify statistically significant differences in the number of GPR17-expressing cells among NC, MD, and NHD brains both in the white matter $(p=0.417)$ and the grey matter $(p=0.545)$ (Figure 5, panels a, b), although NHD1 and NHD3 showed a trend for decrease in the number of GPR17-immunoreactive preoligodendrocytes in inactive demyelinated lesions. In our study, it is impossible to evaluate the levels of endogenous agonists for GPR17, such as uracil nucleotides and CysLTs in paraffin-embedded NHD and control brains.

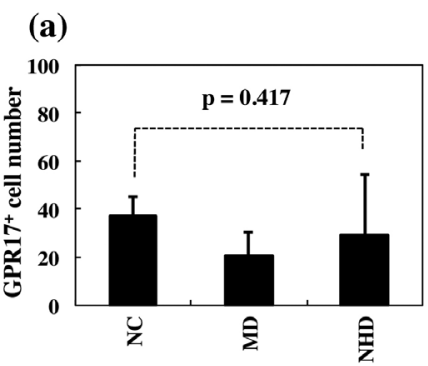

(b)

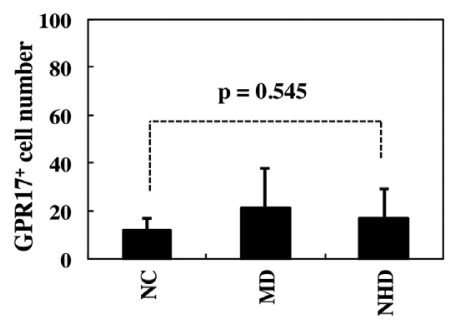

Figure 5. Quantitative analysis of GRP17 expression in the white matter and the grey matter of $\mathrm{NC}, \mathrm{MD}$, and NHD brains. (a) GPR17-positive cell numbers in the white matter of NC, MD, and NHD cases. (b) GPR17-positive cell numbers in the grey matter of NC, MD, and NHD cases. The difference in the average of immunoreactive cell numbers was evaluated by one-way ANOVA with post-hoc Tukey's test.

Importantly, GPR17-expressing OPCs serve as a reserve pool for tissue repair after brain damage (15). GPR17-positive OPCs rapidly react to the damage and undergo maturation in a mouse model of cerebral injury and ischemia (15). The GPR17 agonist UDP-glucose promotes the recovery of myelination in ischemic periventricular leukomalacia (PVL), a rat model of cerebral white matter injury (16), suggesting that GPR17 activation facilitates myelination in vivo. These results suggest that GPR17 plays a beneficial role in remyelination after brain tissue damage. In contrast, in vivo inhibition of GPR17 by GPR17 antagonists or antisense oligonucleotides dramatically reduces ischemic damage in rodent models of focal ischemia, suggesting that GPR17 plays a detrimental role in brain tissue repair $(4,5)$. GPR17 expression is upregulated inside the contused core of brains of the patients with acute traumatic brain injury (TBI), where not only OPCs but also dying neurons, reactive astrocytes, and activated microglia/macrophages express GPR17 (17). Previous studies also showed that neurons surviving in ischemic lesions express GPR17 $(4,5)$. In contrast, we did not find any GPR17-immunoreactive astrocytes, microglia, or neurons in the human brains examined.

In conclusion, we did not find statistically significant differences in the number of GPR17expressing cells distributed in the white matter and the grey matter among NC, MD, and NHD brains. These observations do not support the view that GPR17immunoreactive preoligodendrocytes play a central role in the development of leukoencephalopathy in NHD brains. 


\section{Acknowledgements}

The authors thank Drs. Kenji Jinnai, Nobutaka Arai, Kiyotaka Nakamagoe, Nobutaka Motohashi, and Saburo Yagishita for providing us brain samples. This work was supported by grants from the Research on Intractable Diseases, entitled "Clinicopathological and genetic studies of Nasu-Hakola disease" (H21-NanchiIppan-201; H22-Nanchi-Ippan-136), the Ministry of Health, Labour and Welfare of Japan, and grants from the JSPS KAKENHI (C25430054 and 16K07043) and the Dementia Drug Development Research Center (DRC) project (S1511016), the Ministry of Education, Culture, Sports, Science and Technology (MEXT), Japan.

\section{References}

1. Chen Y, Wu H, Wang S, Koito H, Li J, Ye F, Hoang J, Escobar SS, Gow A, Arnett HA, Trapp BD, Karandikar NJ, Hsieh J, Lu QR. The oligodendrocyte-specific G protein-coupled receptor GPR17 is a cell-intrinsic timer of myelination. Nat Neurosci. 2009; 12:1398-1406.

2. Fumagalli M, Lecca D, Abbracchio MP. CNS remyelination as a novel reparative approach to neurodegenerative diseases: The roles of purinergic signaling and the P2Y-like receptor GPR 17. Neuropharmacology. 2016; 104:82-93.

3. Fumagalli M, Daniele S, Lecca D, Lee PR, Parravicini C, Fields RD, Rosa P, Antonucci F, Verderio C, Trincavelli ML, Bramanti P, Martini C, Abbracchio MP. Phenotypic changes, signaling pathway, and functional correlates of GPR17-expressing neural precursor cells during oligodendrocyte differentiation. J Biol Chem. 2011; 286:10593-10604.

4. Ciana P, Fumagalli M, Trincavelli ML, Verderio C, Rosa P, Lecca D, Ferrario S, Parravicini C, Capra V, Gelosa P, Guerrini U, Belcredito S, Cimino M, Sironi L, Tremoli E, Rovati GE, Martini C, Abbracchio MP. The orphan receptor GPR17 identified as a new dual uracil nucleotides/cysteinyl-leukotrienes receptor. EMBO J. 2006; 25:4615-4627.

5. Lecca D, Trincavelli ML, Gelosa P, Sironi L, Ciana P, Fumagalli M, Villa G, Verderio C, Grumelli C, Guerrini U, Tremoli E, Rosa P, Cuboni S, Martini C, Buffo A, Cimino M, Abbracchio MP. The recently identified P2Y-like receptor GPR17 is a sensor of brain damage and a new target for brain repair. PLoS One. 2008; 3:e3579.

6. Coppi E, Maraula G, Fumagalli M, Failli P, Cellai L, Bonfanti E, Mazzoni L, Coppini R, Abbracchio MP, Pedata F, Pugliese AM. UDP-glucose enhances outward $\mathrm{K}+$ currents necessary for cell differentiation and stimulates cell migration by activating the GPR 17 receptor in oligodendrocyte precursors. Glia. 2013; 61:1155-1171.

7. Simon K, Hennen S, Merten N, Blättermann S, Gillard M, Kostenis E, Gomeza J. The Orphan G Protein-coupled
Receptor GPR17 Negatively Regulates Oligodendrocyte Differentiation via Gai/o and Its Downstream Effector Molecules. J Biol Chem. 2016; 291:705-718.

8. Maekawa A, Balestrieri B, Austen KF, Kanaoka Y. GPR17 is a negative regulator of the cysteinyl leukotriene 1 receptor response to leukotriene D4. Proc Natl Acad Sci U S A. 2009; 106:11685-11690.

9. Fratangeli A, Parmigiani E, Fumagalli M, Lecca D, Benfante R, Passafaro M, Buffo A, Abbracchio MP, Rosa $P$. The regulated expression, intracellular trafficking, and membrane recycling of the P2Y-like receptor GPR17 in Oli-neu oligodendroglial cells. J Biol Chem. 2013; 288:5241-5256.

10. Wolswijk G. Oligodendrocyte precursor cells in the demyelinated multiple sclerosis spinal cord. Brain. 2002; 125:338-349.

11. Klünemann HH, Ridha BH, Magy L, Wherrett JR, Hemelsoet DM, Keen RW, De Bleecker JL, Rossor MN, Marienhagen J, Klein HE, Peltonen L, Paloneva J. The genetic causes of basal ganglia calcification, dementia, and bone cysts: DAP12 and TREM2. Neurology. 2005; 64:1502-1507.

12. Bianchin MM, Capella HM, Chaves DL, Steindel M, Grisard EC, Ganev GG, da Silva Júnior JP, Neto Evaldo S, Poffo MA, Walz R, Carlotti Júnior CG, Sakamoto AC. Nasu-Hakola disease (polycystic lipomembranous osteodysplasia with sclerosing leukoencephalopathy PLOSL): A dementia associated with bone cystic lesions. From clinical to genetic and molecular aspects. Cell Mol Neurobiol. 2004; 24:1-24.

13. Satoh J, Tabunoki H, Ishida T, Yagishita S, Jinnai K, Futamura N, Kobayashi M, Toyoshima I, Yoshioka T, Enomoto K, Arai N, Arima K. Immunohistochemical characterization of microglia in Nasu-Hakola disease brains. Neuropathology. 2011; 31:363-375.

14. Satoh JI, Kino Y, Yanaizu M, Tosaki Y, Sakai K, Ishida T, Saito Y. Expression of gp91phox and p22phox, catalytic subunits of NADPH oxidase, on microglia in NasuHakola disease brains. Intractable Rare Dis Res. 2016; 5:275-279.

15. Viganò F, Schneider S, Cimino M, Bonfanti E, Gelosa $\mathrm{P}$, Sironi L, Abbracchio MP, Dimou L. GPR17 expressing NG2-Glia: Oligodendrocyte progenitors serving as a reserve pool after injury. Glia. 2016; 64:287-299.

16. Mao FX, Li WJ, Chen HJ, Qian LH, Buzby JS. Periventricular leukomalacia long-term prognosis may be improved by treatment with UDP-glucose, GDNF, and memantine in neonatal rats. Brain Res. 2012; 1486:112120.

17. Franke H, Parravicini C, Lecca D, Zanier ER, Heine C, Bremicker K, Fumagalli M, Rosa P, Longhi L, Stocchetti N, De Simoni MG, Weber M, Abbracchio MP. Changes of the GPR17 receptor, a new target for neurorepair, in neurons and glial cells in patients with traumatic brain injury. Purinergic Signal. 2013; 9:451-462.

(Received December 13, 2016; Revised January 30, 2017; Accepted January 31, 2017) 